FACTA UNIVERSITATIS

Series: Mechanical Engineering Vol. 18, $N^{0} 3,2020$, pp. 357 - 373

https://doi.org/10.22190/FUME200305031A

Original scientific paper

\title{
DEVELOPMENT OF A NEW HYBRID MULTI CRITERIA DECISION-MAKING METHOD FOR A CAR SELECTION SCENARIO
}

\author{
Yousaf Ali ${ }^{1}$, Bilal Mehmood ${ }^{2}$, Muhammad Huzaifa ${ }^{2}$, Umair Yasir $^{2}$, \\ Amin Ullah Khan ${ }^{1}$ \\ ${ }^{1}$ Department of Management Sciences, Ghulam Ishaq Khan Institute of Engineering \\ Sciences and Technology, Pakistan \\ ${ }^{2}$ Faculty of Mechanical Engineering, Ghulam Ishaq Khan Institute of Engineering \\ Sciences and Technology, Pakistan
}

\begin{abstract}
Increasing competition in the automobile industry has led to a vast variety of choices when buying a car thus making car selection a tedious task. The objective of this research is to develop a new hybrid multi-criteria decision-making technique, with accuracy greater than that of the already existing methods, in order to help the people in decision-making while buying a car. Hence, considering a broader spectrum, this study aims at easing the process of multi-criteria decision-making problems in different fields. To achieve the objective, seven different alternatives were evaluated with respect to the enlisted evaluation criteria, which were selected after analyzing the secondary data obtained from Pak wheels based on style, fuel economy, price, comfort and performance. These criteria were then analyzed using the proposed Full Consistency Fuzzy TOPSIS method. As the name tells, this method is a unique combination of two techniques. The Full Consistency method is used to calculate the weights of the criteria while the Fuzzy TOPSIS approach is applied to rank the alternatives according to their scores in the selected criteria. The outcomes demonstrate an increase in the consistency ratio of the weight coefficients due to which the ranking of the alternatives by the FCF-TOPSIS is more accurate than the TOPSIS and the Analytical Hierarchy Process. The novelty of the method lies in the fact that this combination has not been used for an alternative selection scenario before. In addition to this, it can be used in various industries where a choice between the available alternatives arises based on a set of evaluation criteria.
\end{abstract}

Key Words: Selection Problems, Car-selection, Decision Problems, MCDM, Fuzzy TOPSIS, FUCOM

Received March 05, 2020 / Accepted August 15, 2020

Corresponding author: Yousaf Ali

Department of Management Sciences, Ghulam Ishaq Khan Institute of Engineering Sciences and Technology, Pakistan

E-mail: Yousafkhan@giki.edu.pk 


\section{INTRODUCTION}

Today cars have become more than just a luxury in the lives of people comparing to the past when they were luxuries that only higher classes could afford while the majority of other people had to rely on conventional transport services. In the $21^{\text {st }}$ century, however, it can be confidently said that the trend is changing. According to the World Bank Collection of Development Indicators, 13 cars are used per 1000 people in Pakistan [1]. This shows that now cars, even though still not accessible to everybody, have become a necessity rather than a luxury. This has been acknowledged by the automobile industries which have now extended their focus from just the rich and powerful to the average middle-class person. Great is a variety of cars to be chosen from that come in all kinds of ranges for people belonging to different backgrounds. It might seem like an easy task to just select a car considering its cost and the desired room space, but due to advancements in technology and an increase in the number of manufacturers, it has become a tedious task. Before someone buys a car, he seeks advice from friends and experts or reexamines the experiences of users of a certain car that can be easily found on credible websites such as Pakwheels and Olx. Mostly, people rank the safety of a car higher while others care about their performance and mileage [2]. With the changing customer demands, car manufacturers have also improved their designs by introducing new technologies such as hybrid and electric cars, i.e. Tesla, Prius, etc. Thus, with a large number of choices, it is relatively harder to select a specific vehicle and that is why it accounts for a complex decision-making situation.

Owing to the sudden increase in car prices along with the increased devaluation of Pakistani currency, the purchasing power of an average person has decreased which means resources are scarce. Thus, it is very important to allocate these resources optimally to achieve maximum satisfaction. The advancement in data sciences and computational problem-solving approaches have led to a vast increase in its scope and made its application in this research possible. In this study, a variety of criteria will be considered such as style, comfort, fuel economy, performance, and price which make it a multi-criteria decisionmaking (MCDM) problem. Furthermore, these five criteria are then subjected to assess seven alternatives, thus giving rise to a decision-making scenario. Such a scenario accounts for proper and effective decision-making tools to make an informed decision. In these situations, Multi-Criteria Decision-Making (MCDM) techniques are taken into account, which have proven to be effective in complex decision-making situations.

Various MCDM techniques have been used previously to solve decision-making problems, the most common being the Analytical Hierarchy Process (AHP) and the Fuzzy AHP. There are a few shortcomings of the AHP which have not been previously emphasized such as its high complexity due to numerous pairwise comparisons; moreover, the results are not precise enough. These limitations are also acknowledged by [3]. According to [4], the AHP technique holds inconsistency when positioning an alternative or a factor as a part of the information set. Similarly, the rank of conservation can be unrealistic when various variations of the AHP methodology are being utilized. Furthermore, using the fuzzy TOPSIS or TOPSIS alone raises issues such as its reliability, meaningful context, and effective results. There are currently many variations of the fuzzy TOPSIS and such variations make it harder for a straightforward selection of a suitable technique [5]. Such limitations count for the development of a new hybrid technique that can produce consistent and reliable results. The requirement of the results being error-free is the need of the hour. 
To counter these problems this paper introduces a new hybrid method by combining a pairwise comparison technique, the Full Consistency Method (FUCOM), and a group decision-making method, the Fuzzy Technique for Order of Preference by Similarity to Ideal Solution (TOPSIS), in the car selection problem. The FUCOM is a relatively new technique for determining the weights of criteria which has never been used before in such a problemsolving. In comparison to other MCDM models, it greatly reduces the mathematical complexity in analyzing the data because of the reduced number of pairwise comparisons. Also, according to Pamucar, it provides a much more precise result than the AHP, which is reflected by a higher degree of consistency in the FUCOM [3]. Similarly, when the criteria weights are calculated using the Fuzzy TOPSIS the deviation of the results from the ideal solution is much higher, as shown by this research. Moreover, while using the AHP, it is assumed that the criteria are independent. If it is not the case, the AHP will calculate inaccurate weights for correlated criteria as the model would no longer be linear. As far as the FUCOM is concerned, the equation model formed is, by default, non-linear, so that it can easily calculate accurate weights for the correlated criteria as well [6]. So, the FCF-TOPSIS accuracy increases further when the accurate FUCOM values are used as an input for the Fuzzy TOPSIS. Other than the increased accuracy this method also gets rid of the problem of rank reversal, which exists in many other MCDM techniques. In this problem, the relative ranks of the alternatives are reversed when an additional alternative is introduced or an existing one is removed. Hence the FCF-TOPSIS can be used to accurately rank any number of alternatives.

Based on the above discussion, the other previous MCDM techniques hold inaccuracies in their results, either alone or in hybrid combination with other techniques. On the other hand, the FUCOM technique proved to be much efficient and reliable in results. To avoid such discrepancies, this study introduces a new hybrid technique known as the FUCOMFuzzy TOPSIS to address the limitations of the previous methodologies. The FCF-TOPSIS is applied to selecting the best possible car as an alternative, based on seven distinct criteria. Apart from this, the study also performs a comparative analysis of two different combinations of techniques, i.e. the AHP-Fuzzy TOPSIS and the fuzzy TOPSIS with that of the FCF-TOPSIS to depict the consistency of the results, thus forming to be the objective of this research study.

The paper proceeds by giving a literature review on the application of Multi-Criteria Decision-Making problems in addition to the scope of the FUCOM and Fuzzy TOPSIS technique; this is followed by a detailed description of the data collection methods, the proposed methodology of the FCF-TOPSIS model and the way of analyzing the data. Furthermore, the results obtained are discussed and the accuracy of the model is verified. The research concludes by listing the outcomes, limitations of this study, and recommendations for further research.

\section{LITERATURE REVIEW}

The society is advancing day by day thus turning the previously considered luxury into a necessity. This is, for instance, the case with motor cars. The possession of cars brings great flexibility to personal mobility. One does not have to worry about the strict schedule of the public transports; instead, he can plan his day according to his routes. Moreover, public 
transport goes along a fixed route which in the case of rush hours can cause delays whereas those using personal cars can change the routes to avoid traffic jams. Furthermore, it is always less comfortable to travel in overcrowded buses/trains; thanks to cars, one does not have to worry about this issue, either [7]. These reasons have influenced people to change their perception and choose cars over buses and other forms of public transport.

Nowadays, there is cut-throat competition in almost everything. Making a decision immediately, i.e. choosing one alternative out of many, is very difficult due to a simple reason that a lot of choices are available in the market with each having its own advantages and disadvantages. While making decisions, people always try to select the most optimal alternative. Such a venture for the optimal solution requires decision-making in complex scenarios which is normally not possible when there are multiple criteria and alternatives to be taken into account [8]. This concept can be related to the objective of this study which requires the selection of an alternative based on five distinct criteria. It involves complex decision-making scenario and thus Multi-Criteria Decision-Making (MCDM) techniques prove to be a feasible solution to such a problem.

Multi-Criteria Decision Methods (MCDM) are widely used as help in making such decision-making processes simpler and selecting the best possible option [9]. MCDM is a technique for evaluating different alternatives based on multiple criteria at a time. It helps the administrator to rank the alternatives according to the qualities of each alternative, and select the worthiest one [10]. A simple example may be of the investors who are in two minds about which projects they should invest in. To address this concern, there is a vast amount of literature dedicated to the selection of the best project alternatives in the past years. One such study discusses various qualitative and quantitative methods of project selection in the paper, namely those which are widely used in the industry today [11].

The scope of the MCDM techniques is not only restricted to large scale projects such as the investor's example given above. It may be used for making daily-life decisions, for example, Sakthivel and IIangkumaran used the Fuzzy Analytical Hierarchy Process (AHP) in the car selection problem [12]. Care should be taken while choosing the criteria based on which the comparison is to be made to obtain accurate results. A study concludes that the selection of the best car model is based on the ranking and efficiency level of the different alternatives [13]. The MCDM applications are not limited; their implementation can be found in various studies and areas of research. One such study that forms the baseline for this research is the selection of hybrid electric vehicles in the scenario of a developing country. The study holds the application of a MCDM technique, i.e. Fuzzy TOPSIS for decision-making application [14]. Similarly, some of latest research studies that incorporate MCDM techniques include: formulation of maintenance framework for the Urea industry [15], using the Fuzzy Lambda-Tau (FLT) for the computation of RAM parameters along with the comparative performance analysis using Fuzzy TOPSIS, Fuzzy EDAS and Fuzzy VIKOR [16], to mention just a few. Other studies that incorporated MCDM techniques include: implementation of fuzzy FMEA and GRA approach for risk assessment of thermal power plant [17], risk failure framework via fuzzy FMEA and EDAS [18], hybrid MCDM techniques such as fuzzy FMEA and GRA for the operational sustainability of the process industry [19], development of meta-model via EDAS [20], to mention just a few.

The study of administrative situations shows that the project selection involves several different criteria; hence it is necessary to evaluate the relative importance of each criterion compared to other ones. This is called assigning a weight to each criterion. The sum of all 
the weights is always equal to one [21]. Different operational techniques are used for this purpose. Mahmoodzadeh and Shahrabi have used the AHP to determine the weights of different criteria used to evaluate different projects [22]. Similarly, to calculate the criteria weights for imprecise or vague data, Fatemeh Torf used the Fuzzy AHP which evaluates data in the form of linguistic variables [23].

The weights of the decision criteria highly affect the results of the MCDM techniques. Thus, the evolution of the available weight determination methods and the formation of more precise methods are very important. Theoretically, in multi-criteria evaluations, both subjective and objective weights are used but in practice, only subjective weights are used commonly [24]. Hence Pamucar developed a new subjective model to determine the weights of criteria which is the Full Consistency Method (FUCOM). It is based on a pairwise comparison of the criteria and the total number of comparisons is $n-1$ where $n$ is the number of criteria. Pamucar also proves in his paper that the FUCOM gives more accurate results, in terms of consistency, as compared to other methods (Best Worst Method (BWM) and AHP) with a reduced number of comparisons. Moreover, the FUCOM is much simpler and more flexible when it comes to the application of the technique. Some of the latest FUCOM technique applications can be seen in the form of selecting a brigade command post, in a hybrid combination with a multi-attributive border approximation area comparison (MABAC) method [25]. Similarly, another study highlights the ranking of dangerous sections of the road via hybrid approach of the FUCOM with the Weighted Aggregate Sum Product Assessment Method (WASPAS) [26]. The same combination of methodology has been utilized in another study for the purpose of selecting a forklift for a warehouse [27]. Furthermore, evaluation of criteria for a sustainable supplier selection is also one of the extensions of FUCOM applications [28]. Another study depicts the FUCOM-WASPAS application for the purpose of the optimization of a wood company's Supply Chain Management (SCM) [29]. Lastly, the FUCOM in combination with another MCDM technique i.e. AHP, possesses application in the ranking of Libyan Airlines [30]. All these applications show that the FUCOM technique has the capability of producing reliable results, both individually and in combination with other techniques.

Some people have used a single technique for the selection of the best possible alternative. Gungor and Isler adopted the Analytical Hierarchy Process for the selection of the best alternative among multiple different alternatives [31]. Dae-Ho Byun has also used an extension of the AHP to decide on an automobile model [2] whereas Mahmoodzadeh and Shahrabi showed how different techniques can be combined for one single task. After calculating the weights for each criterion using the Analytical Hierarchy Process, they used the Fuzzy TOPSIS to determine the best possible alternative. The fuzzy technique was used to allow the incorporation of qualitative, incomplete, and variable data into the decision-making model. Fuzzy Techniques are more accurate when real-life problems are to be solved [22]. Similarly, Tapas and Pritha combined AHP and TOPSIS methods to assist in choosing the best commercially available scooter and then compared their results with the Multi-Attributive Border Approximation Area Comparison method (MABAC) [32].

A common issue that occurs in various MCDM techniques is a rank reversal problem. According to Yong, when a new alternative which was not thought of when the decisionmaking process was initiated, is introduced or an existing one is removed the relative ranks are reversed [33]. Senouci and Mushtaq illustrated the problem of rank reversal in the TOPSIS method [34]. Similarly, Ziemba and Kong demonstrated how this problem affects the results of the Analytical Network Process in their respective papers [35] [36]. 
The selection of the most appropriate car on the basic five distinct criteria via a novel technique of the FUCOM-Fuzzy TOPSIS proves to be the novelty of this study. The previous literature has no application of such a hybrid combination of MCDM techniques. Furthermore, the application of this novel technique is also compared with the existing MCDM techniques; the assumptions based on the effectiveness of this new hybrid technique are drafted. This confirms to be the novelty of this research study.

\section{METHODOLOGY}

Fig. 1 below illustrates the path followed to obtain the results of this research.

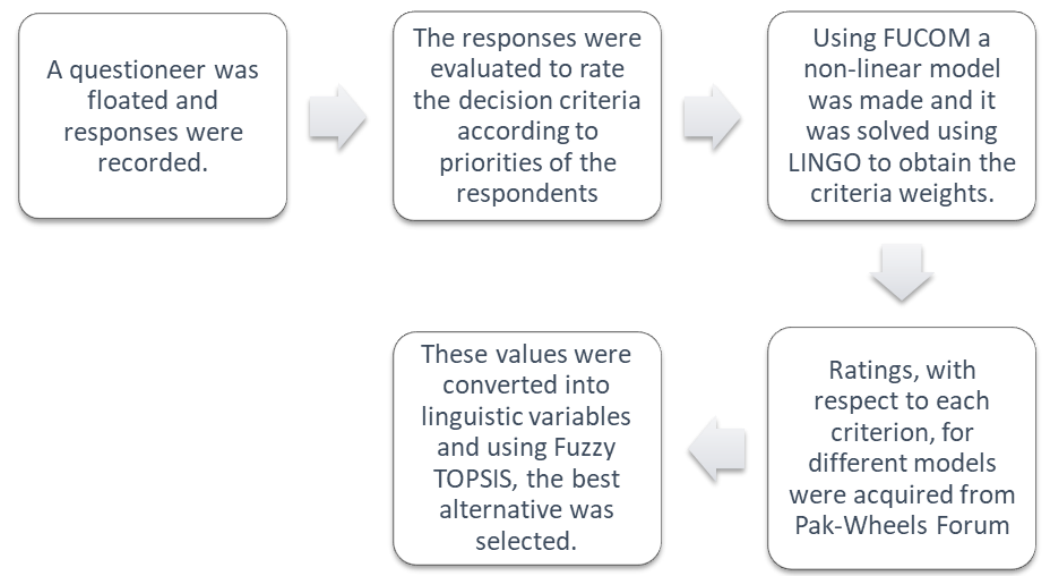

Fig. 1 Flow chart of adopted methodology

\subsection{Data Collection}

This section elaborates on the approach that this paper used to collect the data for the development of the FUCOM Fuzzy TOPSIS (FCF-TOPSIS) technique.

Initially, the criteria were decided upon, i.e., those according to which the comparison of the alternatives was done. For this very purpose, data from the Pak-Wheels website was used to get information about the criteria which are considered important by car owners when they provide feedback. The factors that are mainly discussed in the feedback sections show the five most common and relevant criteria as depicted in Table 1. The list of criteria may differ when the same study is conducted in some other country. Table 1 below lists these criteria. After this, a questionnaire was drafted. It was floated to the car users all over Pakistan, through social media and emails, in which they were asked to rate each criterion, on a scale of 1 to 5 with 1 being least important and 5 being most important one, according to their judgment. A total of 70 responses were collected. This data was used to calculate the absolute rating ( $r$ ) of each criterion by averaging out the responses of each. Next, the most commonly used cars in Pakistan were selected which are A1 - Honda Civic (1.8 i-VTEC), A2 - Honda City (1.3), A3 - Honda BR-V (i-VTEC), A4 - Toyota Corolla GLI (1.3 Automatic), A5 - Toyota Aqua, A6 - Daihatsu Mira and A7 - Toyota Vitz for 
further analysis. Keeping the selected criteria in mind, the performance score for each selected alternative was obtained from numerous reviews present on Pak Wheels. The sample size was different for each alternative. There were 360 reviews for Corolla, 248 for City, 213 for Civic, 57 for BR-V, 34 for Vitz, 12 for Aqua, and 10 for Mira available on the website.

Table 1 List of criteria for comparison of different models

\begin{tabular}{l}
\hline Criteria for comparison of different models \\
\hline C1 - Style \\
C2 - Fuel economy \\
C3 - Price \\
C4 - Comfort \\
C5 - Performance \\
\hline
\end{tabular}

The gathered scores were converted into linguistic variables to incorporate the fuzziness that existed due to varying opinions of the reviewers. For example, the prices of the different models were determined as shown in Table 2. The value for each alternative was then divided by the maximum value. The obtained results, also shown in Table 2, were mapped on the graph shown in Fig. 2, and the linguistic variables were assigned for the price criterion according to its position on it. For instance, the linguistic value obtained for BR-V is 0.871 , as shown in Table 2 below, which lies just before the midvalue of High and Very High region of Fig 2. Thus, being closer to the High region, it was assigned the linguistic variable "High." This was done for all the criteria to assign the linguistic variables. As a result, a comparison matrix of the different alternatives in linguistic terms, as shown in Table 3, was obtained. This matrix was further used in fuzzy TOPSIS to determine the final ranking of the alternatives.

Table 2 Conversion of price into linguistic terms

\begin{tabular}{lcc}
\hline Car & Price & Value \\
\hline A1 - BR-V & 3049000 & 0.8714 \\
A2 - CITY & 2309000 & 0.6599 \\
A3 - COROLLA & 2849000 & 0.8142 \\
A4 - CIVIC & 3499000 & 1.0000 \\
A5 - AQUA & 2600000 & 0.7431 \\
A6 - VITZ & 2300000 & 0.6573 \\
A7 - MIRA & 1690000 & 0.4830 \\
Maximum value & 3499000 & \\
\hline
\end{tabular}

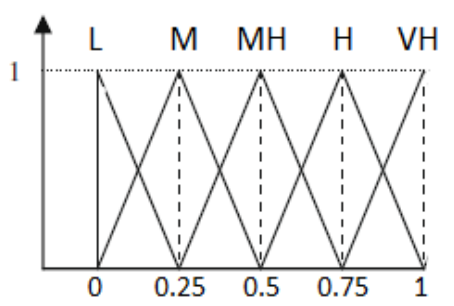

Fig. 3 Linguistic variable graph (author's self-constructed) 
Table 4 Rating cars in terms of linguistic variables.

\begin{tabular}{lccccccc}
\hline Criteria & A1 & A2 & A3 & A4 & A5 & A6 & A7 \\
\hline Price (C2) & VH & H & H & H & H & MH & H \\
Fuel Economy (C2) & H & H & H & MH & VH & VH & H \\
Performance (C3) & H & H & VH & H & VH & H & H \\
Comfort (C4) & H & H & H & H & H & H & VH \\
Style (C5) & VH & H & VH & H & H & H & VH \\
\hline
\end{tabular}

\subsection{FUCOM (Full Consistency Method)}

Determination of criteria weights is one of the important tasks in multi-criteria decision-making. The weights of criteria can greatly affect the outcome of the decision model which is the reason why great attention must be paid to their determination.

The Full Consistency Method is a relatively new method for calculating the criteria weights which were developed by Dragan Pamucar, Zeljko Stevic, and Sinisa Sremac in 2018 [3]. It is considered better than much other multi-criteria decision-making (MCDM) methods because of its lower mathematical complexity thus reducing the chances of errors. The FUCOM method is shown below:

$A_{i}=$ represents the alternatives $(\mathrm{i}=1,2 \ldots \mathrm{b})$

$C_{j}=$ represents the criteria $(\mathrm{j}=1,2 \ldots \mathrm{a})$

$a=$ number of criteria

$b=$ number of alternatives

Step 1:

Firstly, criteria $\mathrm{C}_{\mathrm{j}}$ are ranked according to their ratings, determined after evaluating the questionnaire responses.

$\mathrm{C}_{1}>\mathrm{C}_{2}>\ldots>\mathrm{C}_{\mathbf{L}}$, where $\mathrm{L}$ represents the rank of the criteria $(\mathrm{L}=1,2, \ldots \mathrm{a})$, and if any two criteria have the same significance, ">" is replaced with "=" sign.

Step 2:

Secondly, the ranking criteria are compared using two-way comparisons, and comparative priority $(\alpha)$ is calculated. The comparative priority is the superiority of the criterion of the $\mathrm{C}_{\mathrm{L}}$ rank compared to the criterion of the $\mathrm{C}_{\mathrm{L}+1}$ rank.

These comparative priorities are then represented through a vector.

$$
\beta=\left(\alpha_{1 / 2}, \alpha_{2 / 3 \ldots} \alpha_{L / L+1}\right)
$$

To calculate these priorities, initially, significance $\mathbf{S}_{\mathbf{L}}(\mathrm{L}=1,2,3 \ldots)$ of the highestranked criterion is determined with respect to the rest of the criteria, by using absolute ratings (r) obtained from the questionnaire.

$$
S_{L}=\frac{r_{1}}{r_{L}}
$$

For example, if $C_{1}>C_{2}>C_{3}$ so $S_{2}=(w 1 / w 2)$ and $S_{3}=(w 1 / w 3)$. The value of $S_{1}$ is equal to 1 because it is the highest-rated criterion.

Using the Significance values, the comparative priorities are calculated using the formula:

$$
\alpha \frac{L}{L+1}=\frac{S_{L+1}}{S_{L}}
$$


Next, certain conditions are incorporated to find the weights in Step 3.

Step 3:

Using this information, two conditions are applied to form a non-linear programming model.

The comparative priorities should be equal to the ratio of weights of respective criteria

$$
\frac{w L}{w L+1}=\alpha \frac{L}{L+1}
$$

The condition of mathematical transitivity must be met

$$
\alpha \frac{L}{L+1} \times \alpha \frac{L+1}{L+2}=\alpha \frac{L}{L+1} \text { hence } \frac{w_{L}}{w L+1} \times \frac{w_{L+1}}{w_{L+2}}=\frac{w_{L}}{w_{L+2}}
$$

which leads us to the equation

$$
\frac{w L}{w L+2}=\alpha \frac{L}{L+1} \times \alpha \frac{L+1}{L+2}
$$

If both the conditions are met, the requirement for maximum consistency is met, i.e., deviation in full consistency (DFC) is $\chi=0$. The whole point of the FUCOM method is to minimize DFC for better accuracy in results. Using Eqs. 4 and 6 a non-linear programming model is made for determining the final values of the weight coefficients.

$$
\begin{gathered}
\operatorname{Min} \chi \\
\text { S.t. } \\
\left|\frac{w_{L}}{w_{L+1}}-\alpha \frac{L}{L+1}\right| \leq \chi \\
\left|\frac{w_{L}}{w_{L+1}}-\left(\alpha \frac{L}{L+1} \times \alpha \frac{L+1}{L+2}\right)\right| \leq \chi \\
\sum_{L=1}^{a} w_{L}=1
\end{gathered}
$$

$$
w_{L} \geq 0
$$

By solving this nonlinear model using LINGO Software, the criteria weights can be calculated and the degree of DFC $(\chi)$ is obtained to see whether the results are consistent or not.

\subsection{Fuzzy TOPSIS Method}

TOPSIS stands for the Technique for Order of Preference by Similarity to Ideal Solution which was introduced by Hwang and Yoon in 1980 and is currently one of the most commonly used methods for MCDM problems [37]. In time, changes were made to the originally proposed technique. One such alteration was the incorporation of Zadeh's Fuzzy Set theory to apply the method to a vague data set as well [38]. 
Table 5 Showing the importance weights and ratings for Fuzzy TOPSIS method

\begin{tabular}{lcc}
\hline Linguistic Variables & Importance Weights & Importance Ratings \\
\hline Low & $(0,0.1,0.3)$ & $(0,0,0.25)$ \\
Medium & $(0.3,0.5,0.7)$ & $(0,0.25,0.5)$ \\
Medium High & $(0.5,0.7,0.9)$ & $(0.25,0.5,0.75)$ \\
High & $(0.7,0.9,1.0)$ & $(0.5,0.75,1.0)$ \\
Very High & $(0.9,1.0,1.0)$ & $(0.75,1.0,1.0)$ \\
\hline
\end{tabular}

The following is a step by step procedure on how a problem is solved using Fuzzy TOPSIS approach.

Step 1: Firstly, decide upon a group of decision-makers and explain to them the criteria based on which the selection of the alternatives is to be done. For our case, the selection of a motor vehicle, the data was taken from the Pak wheels website which evaluates the opinion of many end-users and critics based on their reviews. This data was then converted into linguistic variables.

Step 2: The average weight of every criterion is to be calculated so that a singular value can be assigned to each criterion. For our case, this step was omitted as the weights were calculated using the FUCOM technique to fulfill the purpose of this paper, design a hybrid MCDM Method.

Step 3: Next comes the selection of appropriate linguistic variables so that the importance rating of each alternative with respect to every single criterion can be found. The criteria are rated based on the importance ratings given in Table 4 above.

$$
X_{j}=\frac{1}{k}\left(x_{j}^{1}+x_{j}^{2}+x_{j}^{3}+\ldots+x_{j}^{k}\right)
$$

$x_{i j}{ }^{k}$ is the rating of the $k^{\text {th }}$ decision-maker, against alternative $i$ and criteria $j$.

Step 4: Succeeding is the transformation of the linguistic terms into fuzzy triangular numbers and a fuzzy decision matrix is constructed.

$$
\begin{gathered}
A=\left(\begin{array}{ccc}
x_{11} & x_{12} & \ldots x_{1 n} \\
x_{21} & x_{22} & \ldots x_{2 n} \\
x_{31} & x_{32} & \ldots x_{3 n}
\end{array}\right) \\
x_{i j}=\left(a_{i j}, b_{i j}, c_{i j}\right)
\end{gathered}
$$

where $m$ is the number of alternatives and $n$ is the number of criteria and $x_{i j}$ represents the triangular fuzzy number

Step 5: Normalization of the fuzzy decision matrix is done.

$$
\begin{gathered}
R=\left[r_{i j}\right]_{m \times n} \\
r_{i j}=\left(\frac{a_{i j}}{c_{j}^{*}}, \frac{b_{i j}}{c_{j}^{*}}, \frac{c_{i j}}{c_{j}^{*}}\right)
\end{gathered}
$$


where, $c_{j}{ }^{*}$ is the maximum or minimum of $c_{i j}$ depending on whether the criteria is a benefit or cost criteria.

Step 6: Next the weights found using the FUCOM technique were multiplied to calculate the weighted normalized matrix.

$$
u_{i j}=r_{i j} \times W_{j}
$$

Step 7: Moving on, the fuzzy positive ideal solution (FPIS) and fuzzy negative ideal solution (FNIS) represented by $\left(F^{*}\right)$ and $\left(F^{-}\right)$, respectively, is found.

$$
\begin{aligned}
& F^{*}=u_{1}^{*}, u_{2}^{*}, \ldots u_{n}^{*} \\
& F^{-}=u_{1}^{-}, u_{2}^{-}, \ldots u_{n}^{-}
\end{aligned}
$$

where, $u_{j}^{*}=(1,1,1,1)$ and $u_{j}^{-}=(0,0,0)$ for benefit criteria and vice versa for cost criteria's, $j=(1,2, \ldots \mathrm{n})$

Step 8: Next step is to find the distance from the positive and negative ideal solution using the following formulas.

$$
\begin{aligned}
& D^{*}=\sum_{j=1}^{n} d\left(u_{i j}, u_{j}^{*}\right) \\
& D^{-}=\sum_{j=1}^{n} d\left(u_{i j}, u_{j}^{-}\right)
\end{aligned}
$$

where $d$ is representing the distance between two fuzzy numbers and is given by the following formula

$$
d\left(a_{i j}, b_{i j}\right)=\sqrt{\left.\frac{1}{3}\left[a_{1}-b_{1}\right)^{2}+\left(a_{2}-b_{2}\right)^{2}+\left(a_{3}-b_{3}\right)^{2}\right]}
$$

Step 9: Lastly, the closeness factor is calculated for every alternative, using the following formula:

$$
C C=\frac{D^{-}}{D^{*}+D^{-}}
$$

Step 10: Select the alternative having the highest value for the closeness factor.

\subsection{FCF-TOPSIS Model}

This model will be a unique combination of FUCOM and Fuzzy TOPSIS techniques. After deciding upon the criteria based on which the alternatives have to be compared, the most important criterion is to be selected. Next comes the comparison of every criterion with respect to the most important criterion and significance values are to be assigned. These two things can be done either by asking an expert for help or by floating a questionnaire and hence evaluating the collected responses. As far as this study is concerned, the questionnaire approach was used. After doing so, the FUCOM technique is 
applied to construct a non-linear programming model that is solved through LINGO Software to determine the weights of the evaluation criteria.

These weights are used to form the weighted normalized rating matrix of the Fuzzy TOPSIS technique. Next follow steps 3 to 10 given for the Fuzzy TOPSIS technique to select one alternative out of many.

\section{RESULTS AND DISCUSSION}

The collected data was analyzed and used in the calculation of the best alternative, by applying the proposed hybrid method. This section illustrates the calculations done to reach optimal alternatives and discusses the final results.

Based on the responses gathered through the questionnaire, the absolute rating was calculated for each criterion on a scale of 1 to 5 by averaging out the responses of each criterion. The rating in Table 5 shows that the price criteria were considered to be the most important whereas people were least concerned about the style of the car.

After this, the significance (SL) of the most important criterion with respect to the rest of the criteria was determined. It is shown in Table 5 that the higher the value of significance, the lower the importance of that criterion is with respect to the most important one; hence the value for the price is 1 because it is considered to be the most important criterion. This was followed by pairwise comparisons to calculate the comparative priority values $(\alpha)$. These values were further evaluated by applying two different conditions and the following non-linear programming model was constructed.

$\operatorname{Min} \chi$

Subject to the following equations:

$$
\begin{gathered}
\left|\frac{W_{1}}{W_{2}}-1.1325\right| \leq \chi,\left|\frac{W_{2}}{W_{3}}-1.069\right| \leq \chi,\left|\frac{W_{3}}{W_{4}}-1.103\right| \leq \chi,\left|\frac{W_{4}}{W_{5}}-1.078\right| \leq \chi \\
\left|\frac{W_{1}}{W_{3}}-1.211\right| \leq \chi,\left|\frac{W_{2}}{W_{4}}-1.179\right| \leq \chi,\left|\frac{W_{3}}{W_{5}}-1.189\right| \leq \chi \\
W_{1}+W_{2}+W_{3}+W_{4}+W_{5}=1 \\
W_{L} \geq 0
\end{gathered}
$$

This model was solved using the LINGO software, which calculated the finalized criteria weights, which are also summarized in Table 5 below.

Table 6 Finalized criteria weights and the values used for calculating them

\begin{tabular}{lccccc}
\hline Criteria & $\mathrm{C} 1$ & $\mathrm{C} 2$ & $\mathrm{C} 3$ & $\mathrm{C} 4$ & $\mathrm{C} 5$ \\
\hline Rating(r) & 4.53 & 4.00 & 3.74 & 3.39 & 3.14 \\
Significance (SL) & 1.00 & 1.13 & 1.21 & 1.34 & 1.44 \\
Weights (WL) & 0.2408 & 0.2126 & 0.1989 & 0.1803 & 0.1673 \\
\hline
\end{tabular}


The weights show that the trend identified by the absolute rating is correct. People consider the car to be the most important factor in selecting a car whereas the car's comfort and style are least significant.

Apart from the weights, LINGO also calculated the value of DFC $(\chi)$ to be 0.000112 . The low value of DFC highlighted the fact that the deviation of calculated weights from optimal value was negligible.

Then the fuzzy decision matrix was generated by assigning triangular Fuzzy ratings to the data shown in Table 3 according to their respective linguistic variables. Triangular fuzzy numbers are used because the decision-makers find them instinctively easy to use and calculate. Also, using triangular fuzzy numbers is has proven to be an effective way to reduce fuzziness from data. It is also proved in the previous referential literature that mostly in practical situations triangular fuzzy numbers are used [39] [40].

These fuzzy ratings were further evaluated to obtain the final results, summarized in Table 6 below.

Table 7 Final Results obtained

\begin{tabular}{ccccc}
\hline Alternatives & $D j^{*}$ & $D j-$ & $C C j$ & Rank \\
\hline A1 & 3.6284 & 1.3944 & 0.2776 & 6 \\
A2 & 3.6213 & 1.4109 & 0.2804 & 5 \\
A3 & 3.5592 & 1.4647 & 0.2916 & 3 \\
A4 & 3.6744 & 1.3606 & 0.2702 & 7 \\
A5 & 3.5514 & 1.4714 & 0.2929 & 2 \\
A6 & 3.5281 & 1.5022 & 0.2986 & 1 \\
A7 & 3.5624 & 1.4620 & 0.2910 & 4 \\
\hline
\end{tabular}

The results show that A6 - Mira is considered to be the most optimal solution because it obtained the highest value of closeness coefficient $(\mathrm{CC})$, the reason being that it had scored either "High" or "Very High" in all of the benefit criteria (which are intended to be maximized) and it scored the minimum, which is "Medium High", amongst all the alternatives for the cost criteria (which is desired to be minimized). Going by the same analogy, all the alternatives have achieved their ranks according to their respective scores in the benefit and cost criteria.

Furthermore, for comparison, the weights were also calculated using AHP. The results obtained from both the techniques are given in Table 7 below. It can be seen that there is not much difference in values. As far as the accuracy of the results is concerned, the lower consistency ratio of FCF-TOPSIS proves that it is more accurate and the results are closer to the optimal values whereas the consistency ratio (CR) of AHP has a value of 0.000648 which is higher than the value of DFC in FCF-TOPSIS. This shows that the results of AHP deviate more from the optimum value because the higher the value of DFC, the higher deviation in the results will be.

In this research, the values used in the criteria comparison matrix of AHP are not consistent with the Saaty's scale because for a fair comparison with the proposed hybrid method the values had to be similar for both although the questionnaire could have been designed to get the ratings according to the Saaty's scale due to which the results obtained would have shown a further deviated consistency ratio (CR) of AHP. However, our main objective was not to evaluate the data using AHP, but it was just used to prove that FCF- 
TOPSIS is more accurate which is already been proven by the higher CR of AHP. Hence average ratings in decimal numbers were used as inputs to AHP. So, it can be clearly said that the value of AHP's CR was under approximated whereas the calculated DFC $(\chi)$ for the proposed hybrid method is overestimated due to the rounding and approximation of values in the manual calculation; this is the reason why DFC is not equal to zero. Both of these reasons converge on the fact that the actual accuracy of FCF-TOPSIS, in ideal conditions, would be much greater.

Another reason for the higher accuracy of FCF-TOPSIS is that it requires a lower number of pairwise comparisons, $(n-1)$ where $\mathrm{n}$ is the number of criteria, whereas AHP requires $n(n-1) / 2$ a number of pairwise comparisons [3]. This means that for this case, only 4 comparisons had to be done for FCF-TOPSIS whereas AHP needed 10. Due to this the mathematical complexity of FCF-TOPSIS greatly reduces, which in turn decreases the possibility of errors in the result.

Table 8 Comparison of the results of AHP and FUCOM

\begin{tabular}{lcc}
\hline Criteria & $\begin{array}{c}\text { Weights Calculated using } \\
\text { AHP }\end{array}$ & $\begin{array}{c}\text { Weights Calculated using } \\
\text { FCF-TOPSIS }\end{array}$ \\
\hline Price & 0.2407 & 0.2408 \\
Fuel Economy & 0.2123 & 0.2126 \\
Performance & 0.1990 & 0.1989 \\
Comfort & 0.1805 & 0.1803 \\
Style & 0.1671 & 0.1673 \\
Consistency Ratio & 0.0006 & 0.0001 \\
\hline
\end{tabular}

Also, this problem was solved using the Fuzzy TOPSIS and the results were compared with the proposed method. In Table 8, it can be clearly seen that when the value of CR (Consistency Ratio) increases from 0.000112 (FCF-TOPSIS) to 0.000648 (AHP-FUZZY TOPSIS), the closeness coefficient values increase for each respective criterion. Observing the closeness coefficients obtained from FUZZY TOPSIS, the values are much larger than those obtained from FCF-TOPSIS (which had the lowest CR). This shows that, although the same ranks have been obtained, the deviance in the values of FUZZY TOPSIS is larger.

Table 8 Comparison of the closeness coefficient obtained using different techniques

\begin{tabular}{cccc}
\hline Alternatives & FCF-TOPSIS & AHP- FUZZY TOPSIS & FUZZY TOPSIS \\
\hline A1 & 0.2776 & 0.2776 & 0.4706 \\
A2 & 0.2804 & 0.2804 & 0.4800 \\
A3 & 0.2915 & 0.2916 & 0.5069 \\
A4 & 0.2702 & 0.2702 & 0.4511 \\
A5 & 0.2929 & 0.2929 & 0.5125 \\
A6 & 0.2986 & 0.2986 & 0.5297 \\
A7 & 0.2910 & 0.2910 & 0.5045 \\
\hline
\end{tabular}

Hence, the results obtained through the FCF-TOPSIS possess higher accuracy than the Fuzzy TOPSIS. The reason for this is that the Fuzzy TOPSIS technique is applied with the assumption that all the evaluation criteria are independent of each other whereas in practical situations this can never be true. It operates so that the optimal solution is the 
one which has the largest distance from the fuzzy negative ideal solution (FNIS) and the smallest distance from the fuzzy positive ideal solution (FNIS), which increases its efficiency in handling vague data. However, determination of the Closeness Coefficient by using Euclidean Distance, calculated from FNIS and FPIS, neglects the correspondence of the evaluation criteria. Hence the challenge to determine criteria weights while keeping the deviation low. For example, in the car selection criteria, if a car's style is designed with an emphasis on its attractiveness rather than aerodynamics, then its fuel economy will be affected to some extent. This relation is neglected while using TOPSIS but the pairwise comparison technique used in this research, FUCOM, acknowledges it.

Summing up, the proposed method incorporates the advantage of both the pairwise comparison method and the group decision-making technique. As the value of criteria weights obtained is accurate, with negligible deviation from optimal value, the final alternative rankings calculated using FCF-TOPSIS are much precise and have lower mathematical complexity than other MCDM techniques used in this field. In addition to accuracy, the method is not just limited to solving independent criteria, like AHP and TOPSIS, but it can evaluate correlated criteria with the same ease as well. Such results achieve the main objectives of the study, i.e. the selection of the most feasible vehicle in the case of Pakistan and the incorporation ofe the most effective MCDM method by verifying the results in comparison with the other MCDM techniques.

\section{CONCLUSION}

Ease of access to transportation is the need of the hour for almost every individual. With the advancement of technology and the work environment, people need to commute quickly from one place to another. This need calls for a perfect vehicle that can serve the individual according to his or her requirements. For this purpose, this research paper takes five criteria into account based on which seven alternatives are to be assessed. To do so, the study incorporates a hybrid combination of the FUCOM-Fuzzy TOPSIS. After the assessment, the results concluded the Toyota Mira to be the best suitable option for the people living in Pakistan. It is concluded that people who look forward to buying the car can have the best option in the shape of the Toyota Mira while keeping the factors of style, fuel economy, price, comfort and performance in mind.

Furthermore, another objective of this paper was to develop a new Multi-Criteria Decision-Making technique to assist the decision-maker in a car selection scenario which comprises various evaluation criteria. The FUCOM and Fuzzy TOPSIS integration is used in this proposed approach. A case scenario in the light of Pakistan was analyzed and compared with another technique, i.e. the AHP to validate the results. The results show that the proposed approach is better than the AHP since it reduces the mathematical complexity by reducing the number of pairwise comparisons yet giving better results which are shown by its lower consistency ratio. In the pairwise comparison problems, sometimes the criteria are correlated due to which the AHP gives inaccurate weights as the model no longer remains linear, while the FUCOM can easily calculate dependent or independent criteria weights as it is by default a nonlinear model. Hence, it can be concluded that the FUCOM can assess results with minimum possible diversion and thus give concluding reliable results as compared to the rest of the MCDM techniques. 
This research study proves to be beneficial in two aspects. Firstly, it can provide a reasonable solution to the individuals who are looking to buy a vehicle based on the aforementioned criteria. Secondly, the research study has proposed a new hybrid technique with reliable results that can be beneficial for the researchers to implement in future research studies.

Research limitations of this study are mainly based on the time constraint which resulted in a lower number of responses. Secondly, the unavailability of the online resources like Pak wheels, which if present, could have provided a comparative overview of the car users' feedback. For this study, the analysis had to rely on just one source.

For future research on this method, a Software could be designed for the FCF-TOPSIS algorithm which would not only save time and could be utilized for manual calculations, but could also improve the results further by taking exact values for calculations rather than approximations. Also, the attributes of each alternative with respect to the evaluation criteria should be taken from experts in the car field rather than the local people so that the results can become more reliable. Furthermore, incorporation of such a concept in another field of study can open doors for the decision-making via this new hybrid MCDM technique.

\section{REFERENCES}

1. World Bank, 2013, World Development Indicators., Report, Washington.

2. Byun, D.H., 2001, The AHP Approach For Selecting An Automobile. Information \& Management, 38(5), pp. 289-297.

3. Pamučar, D., Stević, Ž., Sremac, S., 2018, A new model for determining weight coefficients of criteria in mcdm models: Full consistency method (FUCOM), Symmetry, 10(9), pp. 1-22.

4. Karthikeyan, R., Venkatesan, K.G.S., Chandrasekar, A., 2016, A comparison of strengths and weaknesses for analytical hierarchy process, Journal of Chemical and Pharmaceutical Sciences, 9(3), pp. 1215.

5. Madi, E.N., Garibaldi, J.M., Wagner, C., 2016, An exploration of issues and limitations in current methods of TOPSIS and fuzzy TOPSIS. In : IEEE International Conference on Fuzzy Systems (FUZZ-IEEE), Vancouver.

6. Ishizaka, A., Nemery, P., 2013, Multi-criteria decision analysis: methods and software. John Wiley \& Sons, New Jersey.

7. Anwar, A.M., 2009, Paradox between Public Transport and Private Car as a Modal Choice in Policy Formulation, Journal of Bangladesh Institute of Planners, 2(1), pp. 71-77.

8. Zhao, H., Li, N., 2016, Optimal siting of charging stations for electric vehicles based on fuzzy Delphi and hybrid multi-criteria decision-making approaches from an extended sustainability perspective, Energies 9(4), pp. 270-286.

9. Bhole, G.P., Deshmukh, T., 2018, Multi Criteria Decision-making (MCDM) Methods and its applications, International Journal for Research in Applied Science \& Engineering Technology (IJRASET), 6(5), pp. 899-915.

10. Lee, WS., 2014, A new hybrid MCDM model combining DANP with VIKOR for the selection of location-real estate brokerage services, International Journal of Information Technology \& Decisionmaking, 13(1), pp. 197-224.

11. Mantel, S.J., Meredith, J., 2003, Project Management: A Managerial Approach. Wiley, New York.

12. Sakthivel, G., Ilangkumaran, M., Nagarajan, G., Raja, A., Ragunadhan, P.M., Prakash, J., 2013, A hybrid MCDM approach for evaluating an Automobile Purchase model, International journal of information and decision sciences 5(1), pp. 50-85.

13. Roy, S., Mohanty, S., Mohanty, S., 2018, An Efficient Hybrid MCDM based Approach for Car Selection. In: International Conference on Research in Intelligent and Computing in Engineering (RICE), San Salvador.

14. Khan, F., Ali, Y., Khan, A.U., 2020, Sustainable hybrid electric vehicle selection in the context of a developing country, Air Quality, Atmosphere \& Health, 13(1), pp. 1-11. 
15. Panchal, D., Chatterjee, P., Shukla, R.K., Choudhury, T., Tamosaitiene, J., 2017, Integrated fuzzy AHPcodas framework for maintenance decision in urea fertilizer industry, Economic Computation \& Economic Cybernetics Studies \& Research, 51(3), pp. 179-196.

16. Panchal, D., Singh, A.K., Chatterjee, P., Zavadskas, E.K., Keshavarz-Ghorabaee, M., 2019, A new fuzzy methodology-based structured framework for RAM and risk analysis, Applied Soft Computing, 74(1), pp. 242-254.

17. Panchal, D., Kumar, D., 2017, Risk analysis of compressor house unit in thermal power plant using integrated fuzzy FMEA and GRA approach, International Journal of industrial and systems Engineering, 25(2), pp. 228-250.

18. Panchal, D., Tyagi, M., Sachdeva, A., 2019, A Novel Framework for Evaluation of Failure Risk in Thermal Power, Springer, Singapore.

19. Panchal, D., Chatterjee, P., Yazdani, M., Chakraborty, S., 2019, A Hybrid MCDM Approach-Based Framework for Operational Sustainability of Process Industry. In : Advanced Multi-Criteria Decisionmaking for Addressing Complex Sustainability Issues. IGI Global, Jalandhar, pp. 1-13.

20. Chatterjee, P., Panchal, D., Chakraborty, S., 2020, A Developed Meta-model for Biomaterials Selection, Trends in Biomaterials \& Artificial Organs, 34(1), pp. 20-32.

21. Enea, M., Piazza, T., 2004, Project Selection by Constrained Fuzzy AHP, Fuzzy optimization and decision-making, 3(1), pp. 39-62.

22. Mahmoodzadeh, S., Shahrabi, J., Pariazar, M., Zaeri, M.S., 2007, Project selection by using fuzzy AHP and TOPSIS technique, World Academy of Science, Engineering and Technology, 30(1), pp. 333-338.

23. Torfi, F., Farahani, R.Z., Rezapour, S., 2010, Fuzzy AHP to determine the relative weights of evaluation criteria and Fuzzy TOPSIS to rank the alternatives, Applied Soft Computing, 10(2), pp. 520-528.

24. Zavadskas, E.K., Podvezko, V., 2016, Integrated Determination of Objective Criteria Weights in MCDM, International Journal of Information Technology \& Decision-making 15(2), pp. 267-283.

25. Bozanic, D., Tešić, D., Milić, A., 2020, Multicriteria decision-making model with Z-numbers based on FUCOM and MABAC model, Decision-making: Applications in Management and Engineering, 3(2), pp. 19-36.

26. Nenadic, D., 2019, Ranking dangerous sections of the road using MCDM model, Decision-making: Applications in Management and Engineering, 2(1), pp. 115-131.

27. Fazlollahtabar, H., Smailbašić, A., Stević, Ž., 2019, FUCOM method in group decision-making: Selection of forklift in a warehouse, Decision-Making: Applications in Management and Engineering, 2(1), pp. 49-65.

28. Durmić, E., 2019, Evaluation of criteria for sustainable supplier selection using FUCOM method, Operational Research in Engineering Sciences: Theory and Applications, 2(1), pp. 91-107.

29. Erceg, Ž., 2019, Integrated MCDM model for processes optimization in supply chain management in wood company, Operational research in engineering sciences: Theory and applications, 2(1), pp. 37-50.

30. Badi, I., Abdulshahed, A., 2019, Ranking the Libyan airlines by using full consistency method (FUCOM) and analytical hierarchy process (AHP), Operational Research in Engineering Sciences: Theory and Applications, 2(1), pp. 1-14

31. Güngör, I., İşler, D.B., 2004, Automobile Selection with Analytical Hierarchy Approach, International Journal of Management Economics and Business, 1(2), pp. 21-33.

32. Biswas, T., Saha, P., 2019, Selection of commercially available scooters by new MCDM method, International Journal of Data and Network Science, 3(2), pp. 137-144.

33. Shin, Y.B., 2017, Rank reversal phenomenon in cross-efficiency evaluation of data envelopment analysis, International Journal of Business and Economic Development (IJBED), 5(1), pp. 35-40.

34. Senouci, M.A., Mushtaq, M.S., Hoceini, S., Mellouk, A., 2016, TOPSIS-based dynamic approach for mobile network interface selection, Computer Networks, 107(1), pp. 304-314.

35. Ziemba, P., Wątróbski, J., 2016, Selected Issues of Rank Reversal Problem in ANP. In : Selected Issues in Experimental Economics, Springer, Cham, pp. 203-225.

36. Kong, F., Wei, W., Gong, J.-H., 2016, Rank reversal and Rank Preservation in ANP, Journal of Discrete Mathematical Sciences and Cryptography, 19(3), pp. 821-836.

37. Hwang, CL., Paidy, S. R., Yoon, K., Masud, A. MS., 1980, Mathematical programming with multiple objectives, Computers \& Operations Research, 7(1-2), pp. 5-31.

38. Zadeh, L.A., 1965, Fuzzy sets, Information and control, 8(3), pp. 338-353.

39. Chang, Y.H., Yeh, C.H., Wang, S.Y., 2007, A survey and optimization-based evaluation of development strategies for the air cargo industry, International Journal of Production Economics, 106(2), pp. 550-562.

40. Xu, Z.S., Chen, J., 2007, An interactive method for fuzzy multiple attribute group decision-making, Information Sciences, 177(1), pp. 248-263. 